\title{
Integration of acoustic and electrical hearing
}

\author{
Christopher Turner, PhD; ${ }^{1-2 *}$ Bruce J. Gantz, MD; ${ }^{2}$ Lina Reiss, PhD $^{1}$ \\ Departments of ${ }^{1}$ Speech Pathology and Audiology and ${ }^{2}$ Otolaryngology-Head and Neck Surgery, University of Iowa, \\ Iowa City, IA
}

\begin{abstract}
For some individuals with severe high-frequency hearing loss, hearing aids cannot provide a satisfactory improvement in speech recognition. However, these same patients often have too much residual hearing to qualify as candidates for a cochlear implant. Here we describe results with the Iowa/ Nucleus Hybrid cochlear implant, which is designed to preserve the patient's residual low-frequency hearing while at the same time supplementing their high-frequency hearing through electrical stimulation. The advantages of this approach are presented, including improved speech recognition in competing backgrounds as compared with traditional cochlear implants. The results with the Iowa/Nucleus Hybrid device demonstrate the ability of the auditory system to integrate acoustic and electrical stimulation, even under conditions of severe distortions to the normal cochlear place-frequency mapping.
\end{abstract}

Key words: cochlear implant, combined acoustic and electrical hearing, frequency selectivity, hearing aid, hearing loss, high-frequency hearing loss, rehabilitation, residual hearing, sensorineural hearing loss, speech recognition.

\section{INTRODUCTION}

Over the past quarter of a century, cochlear implants have become recognized as a highly successful treatment for deafness. The accompanying articles in this issue of $J R R D$ provide ample evidence of the past and current success of electrical stimulation of the inner ear as a treatment for profound hearing loss. However, the majority of persons with hearing loss, including most veterans with hearing loss, are not profoundly deaf; that is, they have some remaining usable hearing. In the present arti- cle, we will update our recent experiences at the University of Iowa in using electrical stimulation of the inner ear via a cochlear implant as a supplement to a patient's existing hearing. In this approach, the goal is to preserve the patient's existing "acoustic" hearing and the "electrical hearing” of the cochlear implant merely adds perception of some of the speech sounds that are missed because of the partial hearing loss.

\section{HIGH-FREQUENCY HEARING LOSS}

The traditional method of treating high-frequency sensorineural hearing loss has been to fit a hearing aid that amplifies the high-frequency regions of speech. With the advent of better hearing aid components in the 1980s, as well as digital technology in the 1990s, it became possible to provide relatively large amounts of gain to these higher frequency regions. However, clinical experience, and some reports in the literature, evidenced that providing

Abbreviations: $\mathrm{CIS}=$ continuous interleaved strategy, $\mathrm{CNC}=$ consonant-nucleus-consonant, $\mathrm{HL}=$ hearing level, $\mathrm{NAL}=$ National Acoustic Laboratories, NIH = National Institutes of Health, SNR = signal-to-noise ratio, SPEAR = speech processing unit for cochlear implants designed with flexibility for research, SRT = speech recognition threshold.

* Address all correspondence to Christopher Turner, PhD; University of Iowa, Department of Speech Pathology and Audiology, 121 SHC, Iowa City, IA 52242; 319-335-8721; fax: 319-335-8851. Email: christopher-turner@uiowa.edu DOI: 10.1682/JRRD.2007.05.0065 
audible speech to high-frequency regions of hearing loss did not always restore speech understanding. Pavlovic [1] and Kamm et al. [2] were some early examples, demonstrating that for some individuals with more severe highfrequency hearing loss, the increase in speech recognition with added high-frequency audibility did not correspond with what would be expected based upon the increase in audible speech information. Hogan and Turner [3] and Ching et al. [4] expanded this idea with more systematic studies, showing that when the hearing loss in the high frequencies (approximately 2,500 $\mathrm{Hz}$ and above) exceeded 60 to $80 \mathrm{~dB}$, some patients exhibited no improvement in speech recognition from high-frequency amplified speech. These results suggest that high-frequency speech cues, which usually involve recognizing consonants, are affected by severe hearing loss to the point where even when amplification is provided, the information transmitted to the brain is insufficient or incorrect because of the loss of sensory cells.

The most common type of sensorineural hearing loss is a loss of hearing sensitivity that increases as frequency increases; this is also known as a "sloping" or "highfrequency" hearing loss. This hearing loss configuration can be linked to many different causes, including aging, noise exposure, and treatment with ototoxic drugs, all of which often occur in the veteran population. In typical high-frequency sensorineural hearing loss, the damage occurs primarily in the basal end of the cochlea, either to the hair cells themselves, or in the case of aging, apparently also to the endocochlear potential that serves to depolarize the hair cells when they are stimulated by sound [5]. When the hearing loss exceeds approximately 60 to $80 \mathrm{~dB}$, this damage to the hair cells begins to affect not only the more susceptible outer hair cells but also results in missing inner hair cells [6]. These inner hair cells are the sensory receptor cells that are responsible for sending signals to the central auditory system; thus, in the case of severe-to-profound hearing loss, information is no longer being transmitted to the brain for the cochlear regions involved. Therefore, electrical stimulation of the auditory nerve, via a cochlear implant inserted only in the base of the cochlea, can provide a means of restoring hearing sensitivity for these high-frequency sounds. This is the basis for the Iowa/Nucleus Hybrid or "short-electrode" cochlear implant developed at the University of Iowa in cooperation with Cochlear Limited (Centennial, Colorado).

\section{COMBINING ACOUSTIC AND ELECTRICAL HEARING ACROSS EARS}

Several authors have reported on the performance of patients who use a traditional long-electrode cochlear implant in one ear and a hearing aid in the contralateral ear [7-9]. In general, the contribution of the contralateral acoustic ear has been shown to be quite helpful, particularly for understanding speech in noise backgrounds. Thus, the general concept of combining acoustic and electrical hearing appears to be a valid and beneficial approach. For many patients with less than profound hearing loss in an ear, however, cochlear implantation is a not an attractive option, particularly if their low-frequency thresholds are in the moderate or better range. The prospect of losing their residual hearing following implantation is a powerful argument against a traditional cochlear implant. The Iowa/Nucleus Hybrid (short-electrode) device was designed specifically to preserve residual low-frequency hearing for this type of patient.

\section{Eligibility}

The most recent clinical trial for the Iowa/Nucleus Hybrid implant recruited patients with the following characteristics. Pure tone thresholds fell within the range shown in Figure 1, which describes a severe-to-profound high-frequency hearing loss for the higher frequencies.

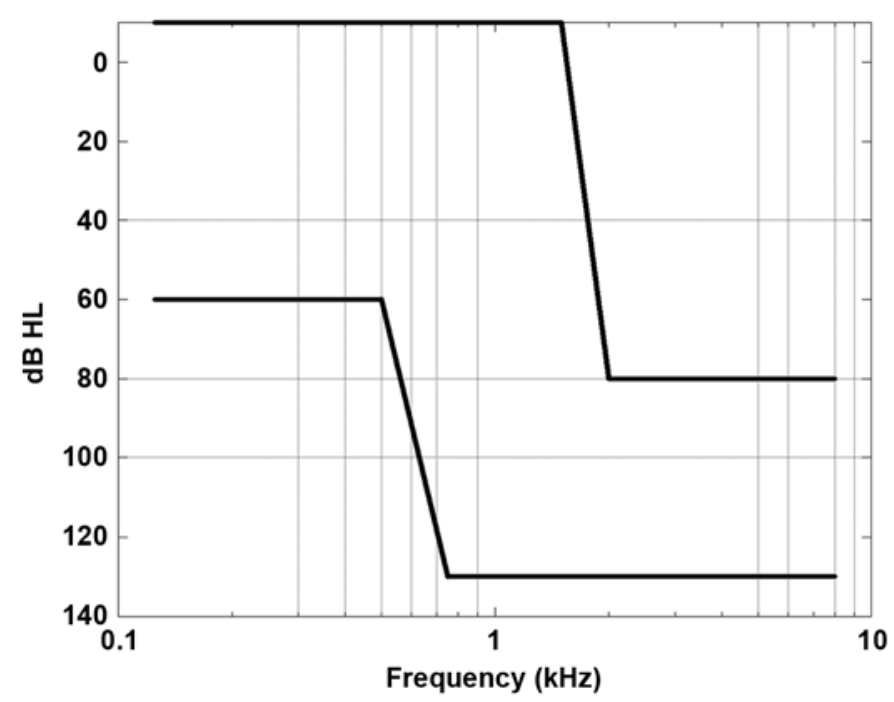

Figure 1.

Eligible pure tone threshold range in implanted ear for Iowa/Nucleus Hybrid candidacy. HL = hearing level. 
The minimum hearing loss for frequencies above $2,000 \mathrm{~Hz}$ was $80 \mathrm{~dB}$ hearing level (HL), which is in agreement with the previously mentioned research demonstrating that amplification for severe high-frequency hearing loss is sometimes not helpful. At present, the assumption is that a traditional long-electrode cochlear implant would be a better choice for candidates with greater than $60 \mathrm{~dB}$ hearing loss in the low frequencies. In the implanted ear, consonant-nucleus-consonant (CNC) word recognition scores lie between 10 and 60 percent, and the contralateral ear can have up to 80 percent correct recognition of CNC words.

\section{Iowa/Nucleus Hybrid Cochlear Implant}

The $10 \mathrm{~mm}$ Iowa/Nucleus Hybrid electrode was designed to be minimally invasive and only enter the descending basal turn of the scala tympani. This short intracochlear electrode has a reduced diameter of $0.2 \times$ $0.4 \mathrm{~mm}$ compared with standard cochlear implants. Six electrodes (channels) are located in the distal $6 \mathrm{~mm}$ of the electrode (Figure 2). How well does implantation of this new electrode preserve residual hearing? In clinical trials to date, residual hearing for the lower frequencies $(<1,500 \mathrm{~Hz})$ has been preserved to within an average of $13 \mathrm{~dB}$ relative to preoperative thresholds in the 80 total subjects who have received this implant. Thresholds at $2,000 \mathrm{~Hz}$ and above have shifted an average of $8 \mathrm{~dB}$. In 96 percent of these ears, some residual hearing has been preserved. The majority (70\%) of threshold shifts are $20 \mathrm{~dB}$ or less.

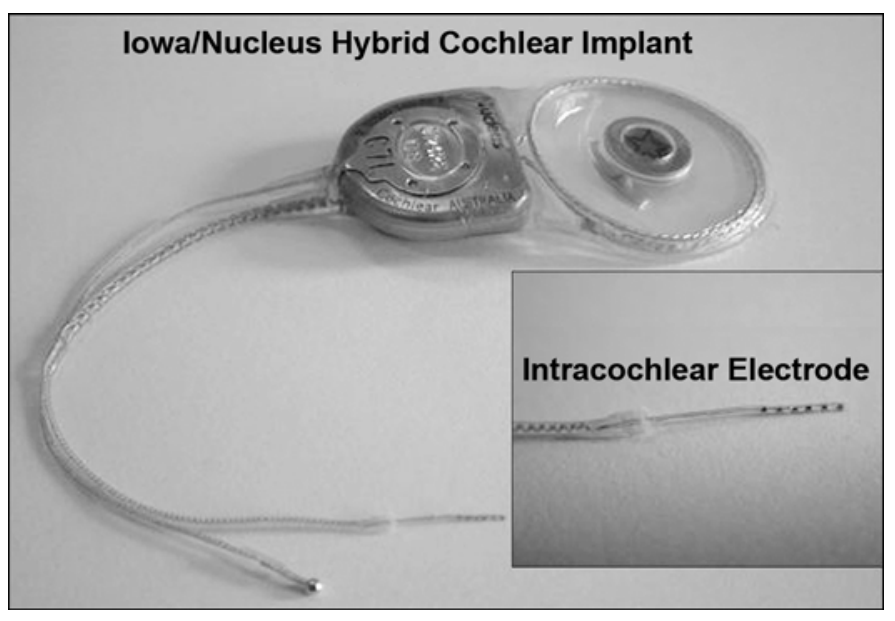

Figure 2.

Iowa/Nucleus Hybrid cochlear implant.

\section{Speech Recognition in Quiet}

The speech recognition performance for Iowa/ Nucleus Hybrid users in quiet backgrounds has been reported in previous publications [10-11]. The performance of the Iowa/Nucleus Hybrid cochlear implant strategy can be viewed several different ways. One view of the Iowa/Nucleus Hybrid device's performance comes from comparing the postoperative acoustic-alone speech recognition performance (implant speech processor turned off, contralateral ear plugged and/or muffed) with the performance obtained when the electrical stimulation is added. In Reiss et al., we reported the most recent results of such a comparison using $/ \mathrm{aCa} /$ consonant speech materials [12]. Some subjects used a hearing aid in the implanted ear depending on their degree of hearing loss (all subjects who wore a hearing aid postoperatively also wore a hearing aid preoperatively as well). If a hearing aid was employed, after implantation it was fit as closely as possible to the National Acoustic Laboratories (NAL) prescription up to the frequency at which the pure tone threshold was $90 \mathrm{~dB}$ or better or $1,000 \mathrm{~Hz}$, whichever was lower. Frequencies above this cutoff were not intentionally amplified; any gain at these higher frequencies was present only in cases where the hearing aid did not allow for an extremely sharp low-pass cutoff. The mean acoustic-alone consonant recognition score was 44 percent correct; after 12 months of implant use, the addition of electrical stimulation increased this score to 58 percent. After 24 months, the score increased to 62 percent. The range of improvements across patients was from 0 to nearly 59 percent. This comparison of postoperative acoustic-alone versus postoperative acoustic plus electrical recognition in the implanted ear demonstrates the effect of adding electrical stimulation to the implanted ear but does not reflect a true preoperative versus postoperative hearing benefit, because, as noted in previous publications, some patients suffered a decrease in hearing thresholds following implantation.

An alternative method of looking at the benefits of the Iowa/Nucleus Hybrid implant is to use a more realworld comparison in which patients are tested when listening with both ears (the implanted ear and the contralateral, acoustic-only ear). Here, preoperative scores are compared with postoperative scores. In these tests, the speech materials were CNC words and the results only included patients implanted and tested at the University of Iowa [9]. Again, some subjects wore a hearing aid in the implanted ear (and also sometimes in the contralateral 
ear) depending on their degree of hearing loss. The preoperative scores represent the "best-aided" condition, with hearing aids set to the standard NAL formula. Following implantation, the hearing aid for the implanted ear was adjusted to amplify only the low frequencies as described previously. The mean preoperative bilateral acoustic-only score was 35 percent; at 12 months after implantation, the mean score was 74 percent with the Iowa/Nucleus Hybrid contribution added, and it remained essentially constant at 73 percent at 24 months. The range of improvements across patients was from 8 to 67 percent.

Clearly, the addition of electrical stimulation for the high-frequency regions of speech for these patients can increase their speech recognition to levels beyond what hearing aids could provide. However, the postoperative speech recognition scores for these patients are not strikingly different from what might be expected of a group of traditional long-electrode patients. One reasonable question to ask then is, "Why not just recommend traditional long-electrode implants for these patients and then not worry about preserving residual acoustic hearing?” The reason is that, in general, residual acoustic hearing is often superior to electrically stimulated hearing in one important quality. This issue will be addressed in the next few sections of this article.

\section{Frequency Selectivity}

Frequency selectivity or frequency resolution refers to the ability of the auditory system to separate sounds based upon their frequency. This ability is impaired in sensorineural hearing loss, as shown in "spread of masking" experiments [13-14] and is indicative of the problems that many hearing-impaired individuals have in understanding speech in noisy backgrounds. Poor frequency selectivity could also be a problem in perceiving the spectral characteristics of some speech sounds. Research presenting speech stimuli in which the frequency resolution is controlled as an independent variable to both cochlear implant subjects and normal-hearing listeners has demonstrated that frequency resolution is a limiting factor for implant users, particularly when the speech is presented in background noise [15-17]. How does the frequency selectivity of cochlear implant users compare with that of normalhearing subjects and subjects with sensorineural hearing loss who still use their acoustic hearing? This question has been answered by Henry et al., who tested all three types of listeners on a spectral-ripple discrimination task [18]. While some amount of overlap was noted between groups, clearly the normal-hearing listeners had the finest resolution, followed by the sensorineural hearing loss group, and in last place were the cochlear implant users. A strong relationship was found between the degree of frequency selectivity and recognition of speech across the entire population $(r=0.82)$. This same strong relationship was also found in subsequent research for the recognition of speech in noise [19]. For this reason, it is worthwhile to consider the potential advantages of preserving residual acoustic hearing (Iowa/Nucleus Hybrid implant) in patients where possible.

\section{EXPERIMENT 1: SPEECH RECOGNITION IN BACKGROUND NOISE}

We measured the ability of Iowa/Nucleus Hybrid listeners to recognize speech in background talk in order to determine what, if any, advantage is gained by preserving residual low-frequency hearing in cochlear implantation. Several previous studies have looked at normal-hearing subjects listening to simulated Iowa/Nucleus Hybrid listening speech [20-21]. In these simulations, the stimuli were processed to have reduced frequency selectivity in the higher frequency regions (cochlear implant-processed speech) and the lower frequency regions were presented in unprocessed form. Both methods have demonstrated a theoretical advantage for providing unprocessed "acoustic" speech to the low-frequency regions of speech in background noise. In Turner et al., three early Iowa/Nucleus Hybrid users were also tested listening to speech in backgrounds and compared with a group of traditional longelectrode cochlear implant users [20]. The long-electrode comparison group was chosen so that their speech scores in quiet were equal to those of the three Iowa/Nucleus Hybrid users. When the background was competing talkers, the signal-to-noise ratio (SNR) advantage for the Iowa/Nucleus Hybrid users over the long-electrode users was $9 \mathrm{~dB}$. In the present report, we expand this comparison to include a much larger group of Iowa/Nucleus Hybrid patients.

\section{Methods}

\section{Subjects}

The subjects for this experiment were all adult users of cochlear implants. The Iowa/Nucleus Hybrid subject group consisted of 19 individuals who had been previously implanted with the $10 \mathrm{~mm}$ Iowa/Nucleus Hybrid 
electrode and had preserved residual low-frequency hearing (within $30 \mathrm{~dB}$ of preoperative values). All wore either the SPrint ${ }^{\mathrm{TM}}$, ESPrit 3G, or Freedom ${ }^{\mathrm{TM}}$ speech processors (Cochlear Limited). Each had worn his or her device for at least 6 months. Their low-frequency residual hearing ranged from mild-moderate hearing loss to severeprofound. Each used the continuous interleaved strategy (CIS) speech processor algorithm for cochlear implants with high-frequency speech (generally above $750 \mathrm{~Hz}$ ) presented through the six electrodes of the $10 \mathrm{~mm}$ electrode (one subject used only five electrodes). The Iowa/ Nucleus Hybrid group mean speech recognition score for a 4-talker, 16-choice consonant recognition test was 62 percent correct (range 32\%-85\%) when they were listening with the acoustic and the electrical hearing in the implanted ear only. When listening with only the electrical hearing, their average score was 44 percent correct. Two additional Iowa/Nucleus Hybrid patients who lost significant amounts of acoustic hearing postoperatively (>30 dB) were also included in some analyses.

The long-electrode group consisted of 20 traditional implant users who had been implanted previously with the Nucleus ${ }^{\circledR} 24$ (Cochelar Limited) (long-electrode) implant and used either the SPrint or ESPrit 3G speech processor. Each had worn his or her device for at least 2 years. They were tested with their own everyday speech processing strategy, which included a mix of advanced combination encoder $(n=10)$, spectral peak speech processor encoder $(n=6)$, and CIS $(n=4)$. Their average recognition score for the same consonant test was 47 percent correct with only the implanted ear. A subgroup of the top-performing individuals was selected from the larger group for some comparisons. This smaller comparison group $(n=11)$ was selected from the larger group in order to match the consonant recognition in quiet abilities of the Iowa/Nucleus Hybrid group. Subjects were chosen starting with the highest performing long-electrode user of the larger group on the same consonant recognition task and proceeding downward until the group mean score matched the Iowa/Nucleus Hybrid group. This top-performing subgroup's mean consonant recognition score (implanted ear only) was 61 percent correct (range 45\%-74\%). Thus, this subgroup closely matched the 62 percent correct consonant recognition scores of the Iowa/Nucleus Hybrid patients.

\section{Procedures}

The target stimuli were spondee words spoken by a female talker and presented in a two-talker background of sentences. Subjects identified the spondees in a 12-alternative closed-set response task. The spondee words were quite easy to recognize for all the implant listeners, and each scored high ( $>90 \%$ correct) for these words when they were presented in quiet; therefore, this task primarily measured the ability of the subjects to resist a noise background. Subjects listened in the sound field with the contralateral ear plugged (and/or muffed), and spondees were presented at $68 \mathrm{~dB}$ sound pressure level. The Iowa/Nucleus Hybrid subjects listened using both their residual acoustic hearing (most wore a hearing aid, but not all) and the electrical stimulation through the short electrode. The level of the background was adaptively varied during the testing procedure until the 50 percent correct SNR was obtained; this value was taken as the speech recognition threshold (SRT) (50\% correct value in $\mathrm{dB}$ SNR) in babble. Full details of the testing procedure are available in Turner et al. [20].

\section{Results}

When the speech in noise performance for the Iowa/ Nucleus Hybrid users was compared with the larger group of long-electrode users, a considerable advantage was noted for the Iowa/Nucleus Hybrid users. The larger $(n=$ 20) long-electrode's group mean SRT was $+6.7 \mathrm{~dB}$, and the Iowa/Nucleus Hybrid's group was $-2.3 \mathrm{~dB}$, for an advantage of $9.0 \mathrm{~dB}$. In this comparison, the "electric-only" speech recognition of the two groups was nearly equivalent (47\% vs $44 \%$ ); however, the larger long-electrode group likely also included subjects with poorer preoperative hearing status than the Iowa/Nucleus Hybrid group; poorer preoperative hearing status has been shown to be a factor in the success of electrical stimulation [22].

The speech in noise performance for the Iowa/Nucleus Hybrid subjects was also compared with the smaller matched group of 11 long-electrode users. As noted previously, the mean SRT for the matched Iowa/Nucleus Hybrid group of 19 subjects was $-2.3 \mathrm{~dB}$, whereas the select longelectrode comparison group's mean was $1.9 \mathrm{~dB}$, for a mean advantage of $4.2 \mathrm{~dB}$. The distribution of individual SRT values for this comparison is displayed in Figure 3. The individual results also show that for a few Iowa/Nucleus Hybrid individuals, SRT values were considerably better than those of any of the long-electrode subjects. Keep in 
JRRD, Volume 45, Number 5, 2008

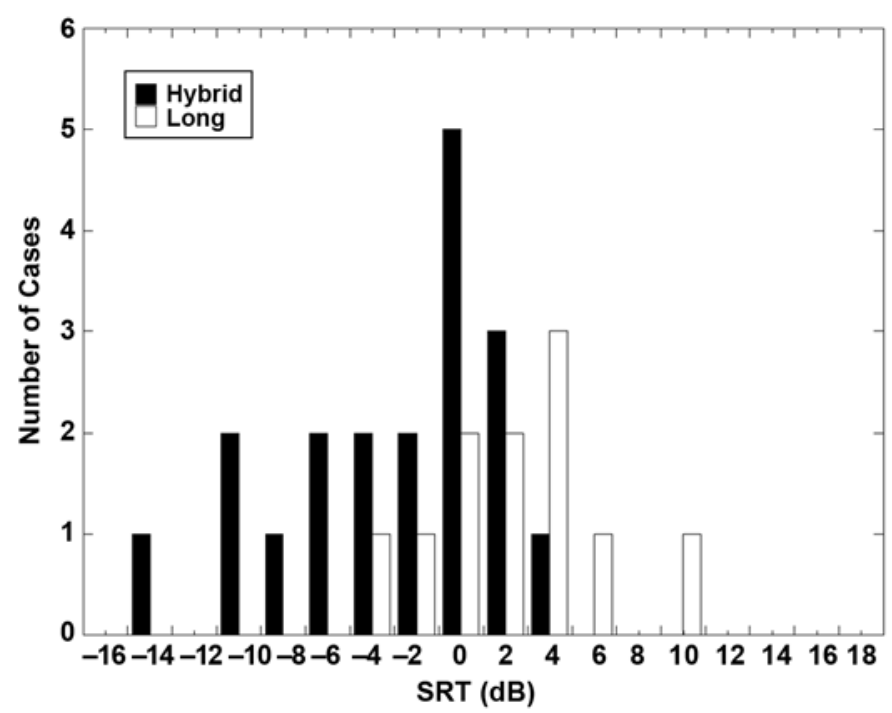

Figure 3.

Distribution of speech recognition threshold (SRT) (50\% correct value in decibel signal-to-noise ratio) values for recognizing spondee words in background of competing talkers for Iowa/Nucleus Hybrid and “matched” traditional long-electrode implant subjects.

mind that these two groups had equivalent speech recognition in quiet (when Iowa/Nucleus Hybrid users were allowed to use both electrical and acoustic hearing). These results further demonstrate the value of preserving residual low-frequency acoustic hearing during cochlear implantation for the recognition of speech in a background of other talkers. The better frequency resolution of the lowfrequency residual acoustic hearing versus electrical stimulation via an implant likely accounts for this advantage. The improved frequency resolution of the preserved residual hearing has also been shown to provide a corresponding advantage in recognizing and appreciating music [23].

Another question of interest is how much residual lowfrequency hearing is required to provide the advantage for speech in background noise over the traditional longelectrode strategy. Does the degree of low-frequency residual hearing determine the ability of the subject to resist the effects of background noise? Kong et al. showed that residual hearing in the contralateral ear could assist cochlear implant users in understanding speech in background noise, even when that contralateral ear was not capable of speech recognition by itself [8]. In Figure 4, the filled squares represent the SRT values for each of the 19 Iowa/Nucleus Hybrid subjects plotted as a function of their pure tone acoustic thresholds (average of 125, 250, and $500 \mathrm{~Hz}$ ). In addition, two open-circle data points are included; these are

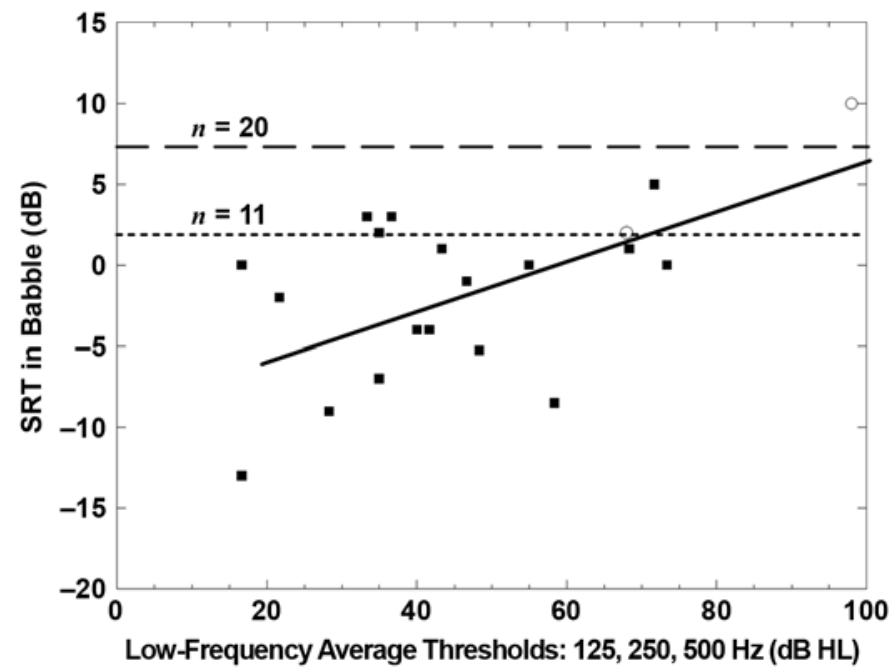

Figure 4.

Relationship between low-frequency average pure tone thresholds and speech recognition threshold (SRT) (50\% correct value in decibel signal-to-noise ratio) values for Iowa/Nucleus Hybrid users. Two data points shown by open circles represent two Iowa/Nucleus Hybrid users who lost significant amounts of residual hearing. Upper dashed line is mean SRT for larger group of long-electrode patients. Lower dashed line is mean SRT for select matched group of long-electrode patients. $\mathrm{HL}=$ hearing level.

two Iowa/Nucleus Hybrid patients whose residual hearing shifted more than $30 \mathrm{~dB}$ following implantation. Looking at just the subjects with preserved residual hearing (filled squares), the relationship between thresholds and SRT is mild ( $r=0.36$, not significant at $p>0.05)$. If the two additional subjects (open circles) are included, the correlation becomes $r=0.56, p<0.05$. Two dashed horizontal lines are displayed in the graph as well. The upper represents the mean SRT value $(+6.7 \mathrm{~dB})$ for the larger group of longelectrode patients $(n=20)$; the lower line represents the mean SRT value $(+1.9 \mathrm{~dB})$ for the select matched group of long-electrode patients shown in Figure $3(n=11)$. A regression line drawn through all the data points suggests that, on average, the advantage of preserving residual hearing exists unless the hearing loss approaches profound levels, although only one data point is present for hearing losses greater than $75 \mathrm{~dB}$ HL. Additional data in this range of hearing loss would be helpful. However, the eligibility criteria for the Iowa/Nucleus Hybrid (Figure 1) do not permit recruiting such patients, and such data will most likely have to come from occasional patients who exhibit a shift in their hearing after implantation or perhaps from long-electrode patients who have some preserved hearing. 


\section{EXPERIMENT 2}

The previous discussion has shown that the auditory systems of the Iowa/Nucleus Hybrid patients are combining acoustic and electrically delivered information for speech understanding. Their acoustic hearing is responsible for speech information only up to approximately $750 \mathrm{~Hz}$, and the electrical hearing provides the remaining (higher) frequencies. This finding is particularly remarkable when one considers that the Iowa/Nucleus Hybrid electrode is only inserted into the very basal end of the cochlea, a region that normally responds to frequencies of 2,800 to $4,700 \mathrm{~Hz}$ and above [24-25]. Thus, the speech information presented through the implanted electrode is "shifted," not only in relation to the normal place-frequency map of the cochlea but also in relation to the acoustically presented information at the lower frequencies, with a potential "gap" in the middle of the cochlea where no information is presented.

The ability of Iowa/Nucleus Hybrid patients to combine acoustic information with shifted electrically delivered information is perhaps not surprising given that previous studies have demonstrated that traditional longelectrode users can adapt over time to an overall shift in the place-frequency mapping when the entire speech frequency range is shifted by changing the speech processor maps [26]. Recent work from our laboratory has also shown that the pitch sensation associated with a particular Iowa/Nucleus Hybrid electrode can shift over time and that this pitch sensation appears to "migrate" toward the frequencies assigned to that electrode by the patient's everyday listening MAP (a cochlear implant speech processor map that assigns speech frequencies to particular electrodes) [27]. In this experiment, we look more closely at the possible effects of assigning "shifted-fromnormal" speech frequencies to the electrodes of the Iowa/ Nucleus Hybrid implant. Two quite different speech processor MAPs were provided to a group of Iowa/ Nucleus Hybrid users. One MAP provided speech that was severely compressed and shifted in cochlear location compared with normal, and the other MAP provided speech that was mainly shifted but not compressed. The patients were allowed to switch between these two MAPS frequently over a period of at least 1 year, after which speech recognition was measured and compared with both MAPS

\section{Methods}

\section{Subjects}

Seven adults users of the Iowa/Nucleus Hybrid device were recruited into this study; each had been using the Iowa/Nucleus Hybrid device (with a standard SPrint processor) for at least 6 months prior to the experiment. Their everyday speech processor MAP up to that point had included all speech frequencies from approximately $750 \mathrm{~Hz}$ to $8,000 \mathrm{~Hz}$ spaced in the default clinical MAP, which uses a semilogarithmic assignment of frequencies across the six electrodes.

\section{Procedures}

These subjects were then fit with an experimental speech processing unit for cochlear implants designed with flexibility for research (SPEAR), which allowed direct control of the frequency bands assigned to each electrode. The SPEAR processor allowed two different MAPs to be stored, and the patient could switch between them easily and at any time. Both MAPs were implemented using the CIS strategy. The two different MAPS were designed to address the mismatch between cochlear place and speech frequencies in two different ways, while at the same time, hopefully, minimizing the difficulties for each patient in understanding speech as they went about their lives. The first MAP, referred to here as "compressed and shifted," assigned a wide range of speech frequencies (essentially everything above $750 \mathrm{~Hz}$ ) to the six basally located electrodes of the Iowa/Nucleus Hybrid. This MAP was therefore essentially the same as the subjects' previous everyday map and was designed with the goal of maximizing the frequency range of speech information presented to the subject.

The second MAP, referred to as "shifted," assigned the speech frequencies from 2,000 to $5,000 \mathrm{~Hz}$ to the Iowa/Nucleus Hybrid electrode, thus maintaining a frequency extent (in octaves) for the speech that approximately matched the "natural" spacing of the six Iowa/ Nucleus Hybrid electrodes. This strategy was chosen in an attempt to still provide adequate speech information to the subjects [28], yet at the same time minimize the shifting and eliminate the severe compression of speech in the first MAP. Figure 5 displays these two strategies schematically. The two SPEAR MAPs are at the top and the bottom of the diagram, and the speech information is located in the center. The hatched areas represent the electrically 


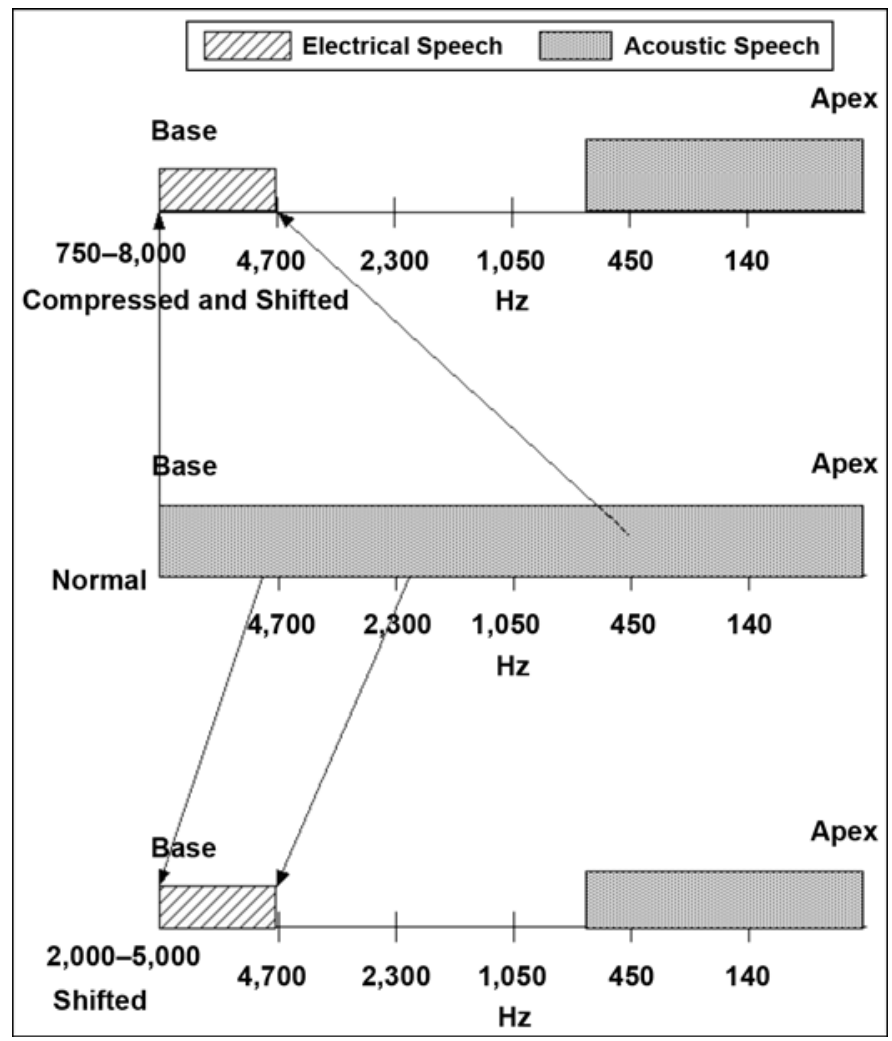

Figure 5.

Schematic diagram of two different frequency allocations to Iowa/ Nucleus Hybrid implant in Experiment 2.

stimulated speech, and the shaded areas represent the acoustic speech. Patients were instructed to switch between the two MAPS often in their everyday life, and all reported that they did so. Speech scores using both MAPs were obtained during regular clinic visits for all patients over a period of at least 1 year, during which time all subjects reached asymptotic and stable performance with each MAP. Subjects were tested with the implanted ear only; the contralateral ear was plugged and muffed for the testing.

\section{Results}

The consonant recognition results for each subject (along with the group mean values) are displayed in Figure 6. While individual differences were noted for four of the seven subjects (three in which "compressed and shifted" was better, one in which "shifted" was better), on average, no clear winner was found between the two very different strategies.

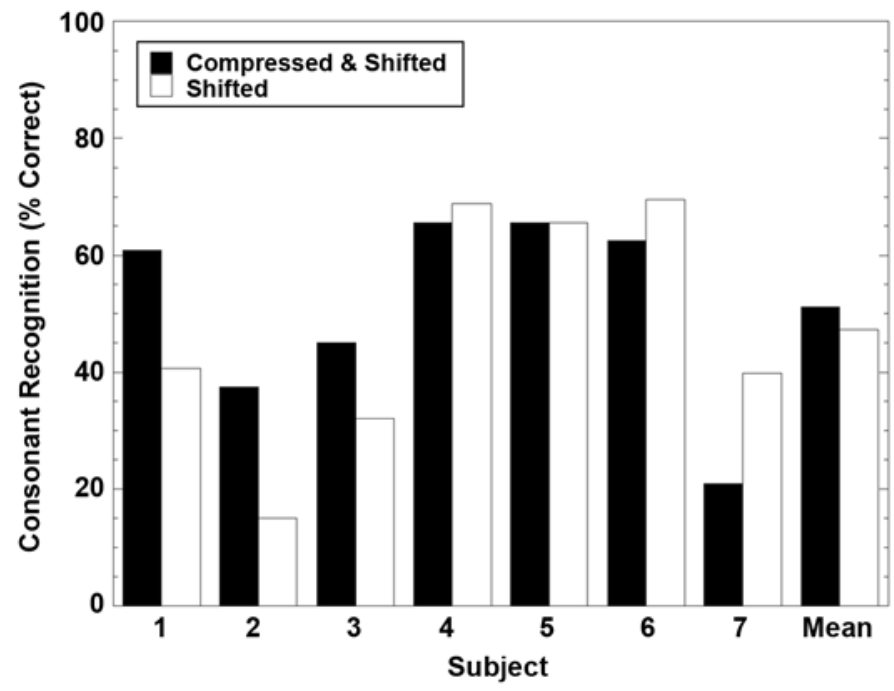

Figure 6.

Individual and group mean scores for consonant recognition for two different frequency allocations in Experiment 2.

The data from Experiment 2, in which no consistent differences were found between the speech recognition abilities of Iowa/Nucleus Hybrid patients as measured under several different types of extreme distortions of the normal place-frequency mapping of the cochlea, add further support to the conclusions of previous researchers. In Fu et al., patients were able to show large improvements with new frequency-place maps over a period of 3 months and at the same time maintain their previous best performance when returned to their previous mapping [26]. In Fu and Shannon, long-term implant users performed best with the map that they had been using for a long period of time, even if that map was distorted relative to the "normal" place-frequency relations of the cochlea [29]. The present study extends these demonstrations of "auditory plasticity" in adult patients to the extreme case, where part of the speech spectrum, the low-frequency acoustic portion, is presented to the "normal" place along the cochlea and the electrically presented speech is shifted (and even severely compressed) to a much more basal location than normal. These findings are also in line with the observations of Reiss et al. that the sensations of pitch can change over time in these patients, perhaps driven by the new place-frequency mapping of electrical stimulation [27]. This flexibility of the auditory system to integrate acoustic and electrical information under conditions of distortion and shifting certainly contributes to the success of the Iowa/ Nucleus Hybrid device. 


\section{SUMMARY}

Combining low-frequency residual acoustic hearing with electrical stimulation for the high frequencies, as in the Iowa/Nucleus Hybrid implant, has some advantages that appear to combine the best of acoustic and electrical hearing (or at least to minimize the disadvantages of either used alone), and this may be an appropriate strategy for more patients in the future. In particular, the advantages of implantation may extend to some patients serviced by the Department of Veterans Affairs with typical audiogram configurations. For example, severe high-frequency hearing loss resulting from blast trauma may benefit from this approach. The development of less-invasive electrodes for all cochlear implants, which might encourage preservation of usable residual hearing, appears to be a desirable future goal as well. In addition, the apparent plasticity of the auditory system to adapt to distortions and shifts in the normal place-frequency mapping may lead to some new developments regarding the optimum length of electrodes in the future.

\section{ACKNOWLEDGMENTS}

This material was based on work supported (in part) by the National Institutes of Health (NIH), National Institute on Deafness and Other Communication Disorders (grants 1 R01 DC000377 and 2 P50 DC00242); NIH, National Center for Research Resources, General Clinical Research Centers Program (grant RR00059); the Iowa Lions Sight and Hearing Foundation; and Cochlear Limited (who developed a cochlear implant to our specifications and provided the devices at no cost).

Cochlear Limited did not have any involvement in the study design; data collection, analysis, or interpretation; and writing or submission of this article.

The authors have declared that no competing interests exist.

\section{REFERENCES}

1. Pavlovic CV. Use of the articulation index for assessing residual auditory function in listeners with sensorineural hearing impairment. J Acoust Soc Am. 1984;75(4):1253-58. [PMID: 6725776]
2. Kamm CA, Dirks DD, Bell TS. Speech recognition and the Articulation Index for normal and hearing-impaired listeners. J Acoust Soc Am. 1985;77(1):281-88. [PMID: 3973220]

3. Hogan CA, Turner CW. High-frequency audibility: Benefits for hearing-impaired listeners. J Acoust Soc Am. 1998; 104(1):432-41. [PMID: 9670535]

4. Ching TY, Dillon H, Bryne D. Speech recognition of hearing-impaired listeners: Predictions from audibility and the limited role of high-frequency amplification. J Acoust Soc Am. 1998;103(2):1128-40. [PMID: 9479766]

5. Schmiedt RA, Lang H, Okamura HO, Schulte BA. Effects of furosemide applied chronically to the round window: A model of metabolic presbyacusis. J Neurosci. 2002;22(21): 9643-50. [PMID: 12417690]

6. Liberman MC, Dodds LW. Single-neuron labeling and chronic cochlear pathology. III. Stereocilia damage and alterations of threshold tuning curves. Hear Res. 1984;16(1): 55-74. [PMID: 6511673]

7. Dooley GJ, Blamey PJ, Seligman PM, Alcantara JI, Clark GM, Shallop JK, Arndt P, Heller JW, Menapace CM. Combined electrical and acoustical stimulation using a bimodal prosthesis. Arch Otolaryngol Head Neck Surg. 1993;119(1): 55-60. [PMID: 8417744$]$

8. Kong YY, Stickney GS, Zeng FG. Speech and melody recognition in binaurally combined acoustic and electric hearing. J Acoust Soc Am. 2005;117(3 Pt 1):1351-61. [PMID: 15807023]

9. Ching TY, Incerti P, Hill M, Van Wanrooy E. An overview of binaural advantages for children and adults who use binaural/bimodal hearing devices. Audiol Neurootol. 2006;11 Suppl 1:6-11. [PMID: 17063004]

10. Gantz BJ, Turner C. Combining acoustic and electric speech processing: Iowa/Nucleus hybrid implant. Acta Otolaryngol. 2004;124(4):344-47. [PMID: 15224850]

11. Gantz BJ, Turner C, Gfeller KE. Acoustic plus electric speech processing: Preliminary results of a multicenter clinical trial of the Iowa/Nucleus Hybrid implant. Audiol Neurootol. 2006;11 Suppl 1:63-68. [PMID: 17063013]

12. Reiss LA, Gantz BJ, Turner CW. Cochlear implant speech processor frequency allocations may influence pitch perception. Otol Neurotol. 2008;29(2):160-67. [PMID: 18025998]

13. Trees DA, Turner CW. Spread of masking in normal subjects and in subjects with high-frequency hearing loss. Audiology. 1986;25(2):70-83. [PMID: 3707439]

14. Glasberg BR, Moore BC. Auditory filter shapes in subjects with unilateral and bilateral cochlear impairments. J Acoust Soc Am. 1986;79(4):1020-33. [PMID: 3700857]

15. Fu QJ, Shannon RV, Wang X. Effects of noise and spectral resolution on vowel and consonant recognition: Acoustic and electric hearing. J Acoust Soc Am. 1998;104(6):3586-96.

[PMID: 9857517] 
16. Friesen LM, Shannon RV, Baskent D, Wang X. Speech recognition in noise as a function of the number of spectral channels: Comparison of acoustic hearing and cochlear implants. J Acoust Soc Am. 2001;110(2):1150-63. [PMID: 11519582]

17. Qin MK, Oxenham AJ. Effects of simulated cochlearimplant processing on speech reception in fluctuating maskers. J Acoust Soc Am. 2003;114(1):446-54. [PMID: 12880055]

18. Henry BA, Turner CW, Behrens A. Spectral peak resolution and speech recognition in quiet: Normal hearing, hearing impaired, and cochlear implant listeners. J Acoust Soc Am. 2005;118(2):1111-21. [PMID: 16158665]

19. Henry BA, Turner CW. The role of spectral resolution in cochlear implant speech recognition in competing backgrounds. In: Proceedings of the 2005 Conference on Implantable Auditory Prostheses; 2005 Aug; Asilomar (CA).

20. Turner CW, Gantz BJ, Vidal C, Behrens A, Henry BA. Speech recognition in noise for cochlear implant listeners: Benefits of residual acoustic hearing. J Acoust Soc Am. 2004;115(4):1729-35. [PMID: 15101651]

21. Qin MK, Oxenham AJ. Effects of introducing unprocessed low-frequency information on the reception of envelopevocoder processed speech. J Acoust Soc Am. 2006;119(4): 2417-26. [PMID: 16642854]

22. Rubinstein JT, Parkinson WS, Tyler RS, Gantz BJ. Residual speech recognition and cochlear implant performance: Effects of implantation criteria. Am J Otol. 1999;20(4): 445-52. [PMID: 10431885]
23. Gfeller K, Olszewski C, Turner C, Gantz B, Oleson J. Music perception with cochlear implants and residual hearing. Audiol Neurootol. 2006;11 Suppl 1:12-15.

[PMID: 17063005]

24. Stakhovskaya O, Sridhar D, Bonham BH, Leake PA. Frequency map for the human cochlear spiral ganglion: Implications for cochlear implants. J Assoc Res Otolaryngol. 2007;8(2):220-33. [PMID: 17318276]

25. Greenwood DD. A cochlear frequency-position function for several species-29 years later. J Acoust Soc Am. 1990;87(6):2592-2605. [PMID: 2373794]

26. Fu QJ, Shannon RV, Galvin JJ 3rd. Perceptual learning following changes in the frequency-to-electrode assignment with the Nucleus-22 cochlear implant. J Acoust Soc Am. 2002;112(4):1664-74. [PMID: 12398471]

27. Reiss LA, Turner CW, Erenberg SR, Gantz BJ. Changes in pitch with a cochlear implant over time. J Assoc Res Otolaryngol. 2007;8(2):241-57. [PMID: 17347777]

28. Lippman RP. Accurate consonant perception without midfrequency speech energy. IEEE Trans Speech Audio Proc. 1996;4(1):66-69.

29. Fu QJ, Shannon RV. Recognition of spectrally degraded and frequency-shifted vowels in acoustic and electric hearing. J Acoust Soc Am. 1999;105(3):1889-1900. [PMID: 10089611]

Submitted for publication May 2, 2007. Accepted in revised form March 4, 2008. 